\title{
From materials characterisation to pre-production validation; the role of the research centre in enabling new approaches to providing processing solutions to industry
}

\author{
Paul L. Blackwell ${ }^{\mathrm{a}}$ \\ Advanced Forming Research Centre, University of Strathclyde, PA4 9LJ, UK
}

\begin{abstract}
Centres such as the AFRC are targeted at bridging the gap between fundamental University research and the needs of industry. The paper describes some of the elements in the process of translating the products of basic scientific research into useful outcomes for industrial manufacturing companies within the metal shaping sector. This commences with a sound knowledge of material mechanical and physical properties within the relevant forming or forging window. This data will then generally be incorporated into a finite element based process model. More sophisticated models will facilitate the prediction of microstructural development during and after forming. However, such models generally still require validation, and in order for such validation to be reflective of industrial practice then full scale or close to full scale trials may be carried out. The AFRC has a range of industrial scale manufacturing equipment which allows such validation to be performed. The net effect of this is that from a manufacturer's point of view a new process may be significantly de-risked prior to introduction into a production environment. The paper will examine some of the approaches used, with specific reference to some of the specialised testing and processing equipment used to translate research into outputs useful to industry.
\end{abstract}

\section{Introduction}

In 2010 the UK Government commissioned Dr Hermann Hauser to examine The Current and Future Role of Technology and Innovation Centres in the UK. The ensuing report examined the challenges involved in translating the excellent basic research carried out in UK Universities into "commercial propositions that can attract venture capital investments or (that could) be licenced" [1]. The report identified the need for a nationally organised "translational infrastructure" that could bridge between research and technology commercialisation. Having reviewed what a range of other countries had done to address this issue Hauser recommended the setting up of a number of Technology Innovation Centres (TICs) whose aim was to exploit a range of targeted technologies and develop them into commercial propositions. A range of focus areas were identified including satellite communications, fuel cells and, of most pertinence here, Advanced Manufacturing.

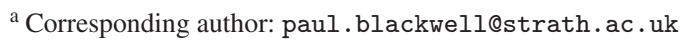

This is an Open Access article distributed under the terms of the Creative Commons Attribution License 4.0, which permits unrestricted use, distribution, and reproduction in any medium, provided the original work is properly cited. 


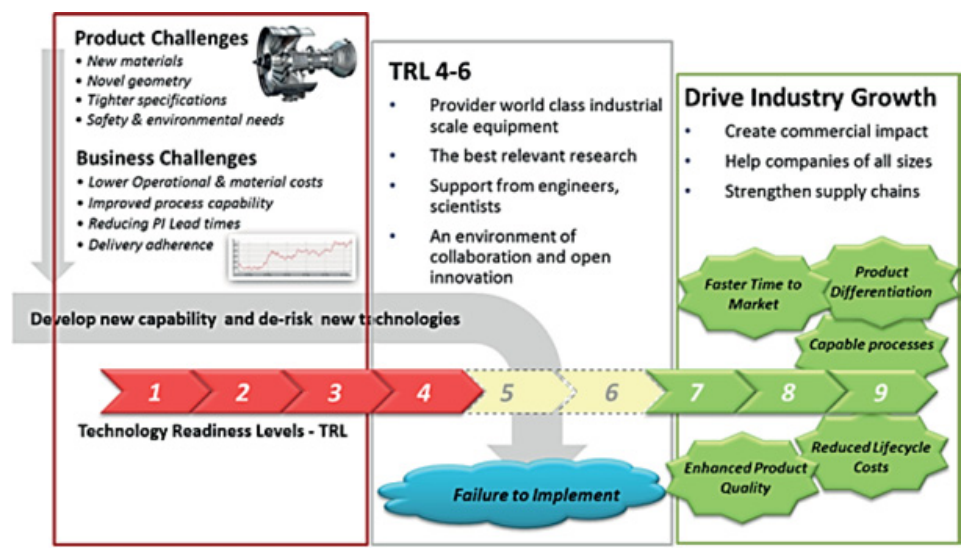

Figure 1. The TRL scale; centres such as the AFRC bridge the gap been University led research and the needs of industry.

Many of the original TICs have now been re-branded as Catapult Centres; the message being that research is being rapidly propelled via such centres into commercialisation. Seen diagrammatically the situation may be represented as shown in Fig. 1. The figure shows the technology readiness spectrum - basic traditional university research would fall within levels 1 to 3 . Prototype demonstration into an industrial environment would fall at level 7, with full productionisation at levels 8 and 9. The Catapult centres occupy the middle ground between 4 and 7 and fulfil the role of the translational infrastructure referred to by Hauser. Here, the focus will be on the work of the High Value Manufacturing (HVM) Catapult and particularly the Advanced Forming Research Centre (AFRC) based here in Glasgow.

The HVM Catapult is a nationally distributed set of research centres within the UK that address a wide variety of industrial challenges. The primary focus of the various centres is listed below:

- AFRC - Forging and forming

- AMRC - Machining

- NAMRC - Nuclear sector

- $\mathrm{CPI}$ - Chemical processing

- NCC - Carbon fibre composites

- WMG - Automotive

- MTC - Welding, automation, tooling and fixtures.

The various centres have a variety of funding mechanisms though in most cases there is a mixture of Government funding, regional funding, commercial research and development and direct funding. The latter is often obtained via a membership model. In the case of the AFRC, the centre is primarily supported by a number of UK and internationally based OEMs and Tier 1 suppliers mainly, though not exclusively, drawn from the aerospace sector. This is supported by a secondary level of membership which mainly consists of equipment or software suppliers e.g. of finite element modelling platforms.

\section{Methodology}

For the concept of a translational infrastructure to work the basic elements have to be available for development. Such basic elements may take a variety of forms e.g.:

- The development or improvement of processing technologies.

- New alloys - or alloy variants. In this case the optimum processing window may need evaluation and definition. By working with a Centre such as AFRC a material supply company can generate a 


\section{ICNFT 2015}

reliable database that it can draw on when customers enquire with regard to optimised processing conditions.

- The development of modelling approaches that are then validated at real scale.

- Developments in measurement/testing. In this case it may be that the Centre acts to demonstrate the applicability of a new sensor type e.g. for NDE, or facilitates the development of new methodologies in geometric measurement which can be tested under simulated production conditions. This allows industrial customers to try out such technologies before they invest themselves - it de-risks the investment.

In practice, we need a number of such elements to be able to deliver the service industry wants. The first aspect of this is that we are processing a material - in our case a metallic alloy - to form some final component / product that has to have a certain geometry and an accompanying property set. In order to optimise the processing we need a sound knowledge of the material properties during the process cycle. This will include both the mechanical (e.g. stress / strain behaviour) and physical properties (e.g. specific heat capacity, thermal conductivity) of the material and a knowledge of the boundary conditions (e.g. temperature, heat transfer coefficient, effect of lubricant). Some of this may be available from the literature, but in many cases it is necessary to generate such data directly. This may involve the use of bespoke equipment.

One of the major outputs of the AFRC is validated process models. What does this mean? Previously many customers were happy if the model was able to predict processing load requirements (e.g. what sized press is required) and indicate basic metal flow e.g. within a closed die forging. Through the latter it becomes possible to evaluate the likelihood of the formation of laps, cracks, or other defects associated with the forming or forging process. There are now available a wide selection of Finite Element (FE) based process models that would claim to do this. However, customers are becoming more demanding and the call now is for process models that can do all of the above but which can also predict microstructural development e.g. such factors as grain growth, recrystallization or texture development. On top of this there is also a strong industry driver to be able to predict, and thus potentially control, the development of residual stress within the material during forging or forming. As is well known, depending on the nature and distribution of such stresses they may enhance or seriously undermine fatigue life. High residual stresses also create problems with geometric distortion during post-forging heat treatments or finish machining operations.

Good process models depend on a number of factors:

- A clear understanding of the process itself e.g. the temperature of workpiece and tooling, the dynamics of the forming equipment (strain rate, elastic deflections within the tooling and the forming equipment itself), in some cases e.g. incremental forming processes such as flow forming, the stress state at the tooling/workpiece interface.

- A robust constitutive model that describes material behaviour within the deformation space (temperature, strain rate, strain) that the process is operating under.

- A clear understanding of the boundary conditions.

- A good FE code and someone with the necessary knowledge to understand its limitations and strengths.

\section{Examples}

\section{Specialist equipment}

One of the advantages of centres such as the AFRC is that they have the necessary funding to support the development of bespoke equipment. Figure 2 shows a high rate test machine commissioned by the AFRC specifically designed to generate mechanical property data at deformation rates of up to 3 metres per second. This $500 \mathrm{kN}$ machine, heated by induction, allows data to be generated in the temperature 


\section{MATEC Web of Conferences}

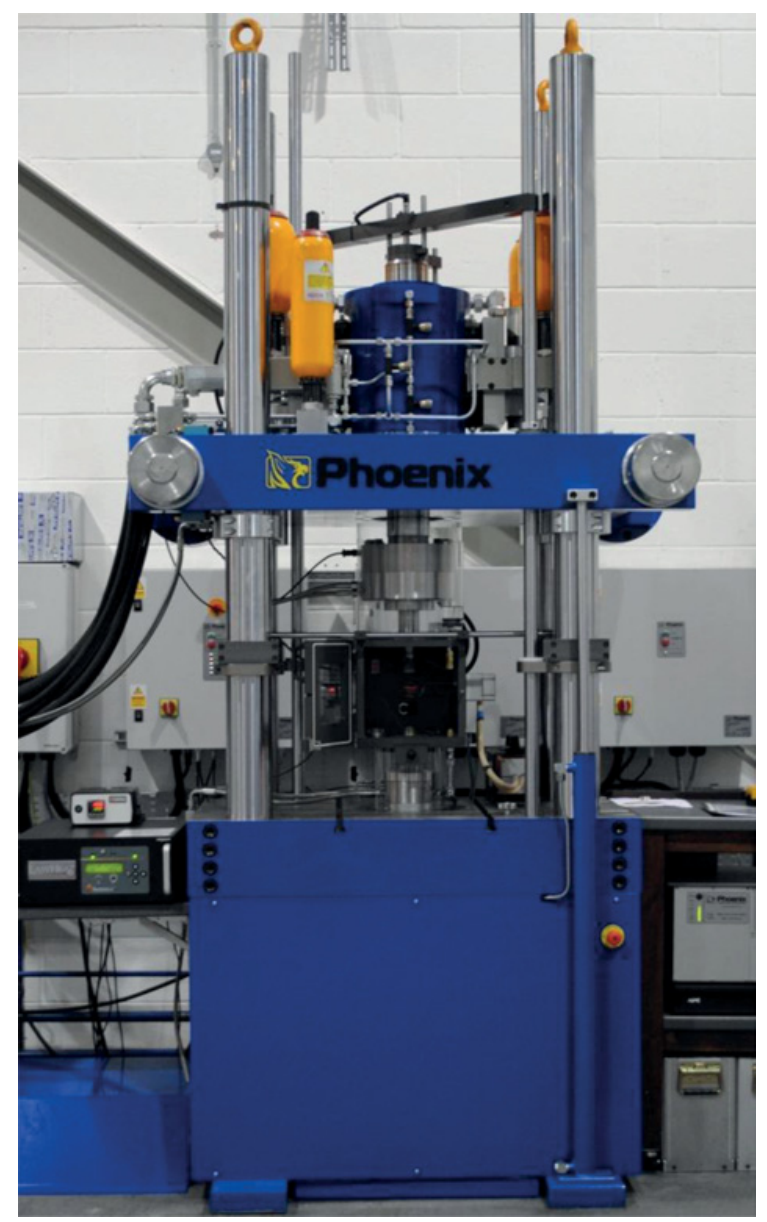

Figure 2. High rate compression testing machine used for obtain baseline data for forging models.

and strain rate range that is typical for hammer and screw presses. Conventional hydraulic test machines struggle to match the speed and control requirement offered by this equipment. The data generated by equipment such as this is used in forging process models.

Figure 3 shows one of the AFRC forging presses. This press, designed by Schuler, features a 500T vertical and 350T horizontal action. Such double action presses are often used for the manufacture of preforms prior to final forging. The novel feature of this press is the inclusion of two servo-drive motors that give direct and accurate control of the movement of the rams. Conventional multiforges are mechanically driven and rely on inertial effects. The result of this is that the ram tends to slow down towards the end of the stroke. This can compromise results, particularly with some of the more difficult to forge alloys that are prone to cracking during forging. Recent work at the AFRC has demonstrated that the extra control of the servo-driven press can enable the forging of alloys that are difficult to forge on conventional presses.

Figure 4 shows a rotary forge. This particular example was custom designed for the AFRC. The concept of rotary forging is of course not new, however, this technology is not widely used in the UK. Part of the AFRC's mission is to prove the capability of rotary forging on a new generation of alloys and to develop a range of products through to the prototype stage so that interested companies 


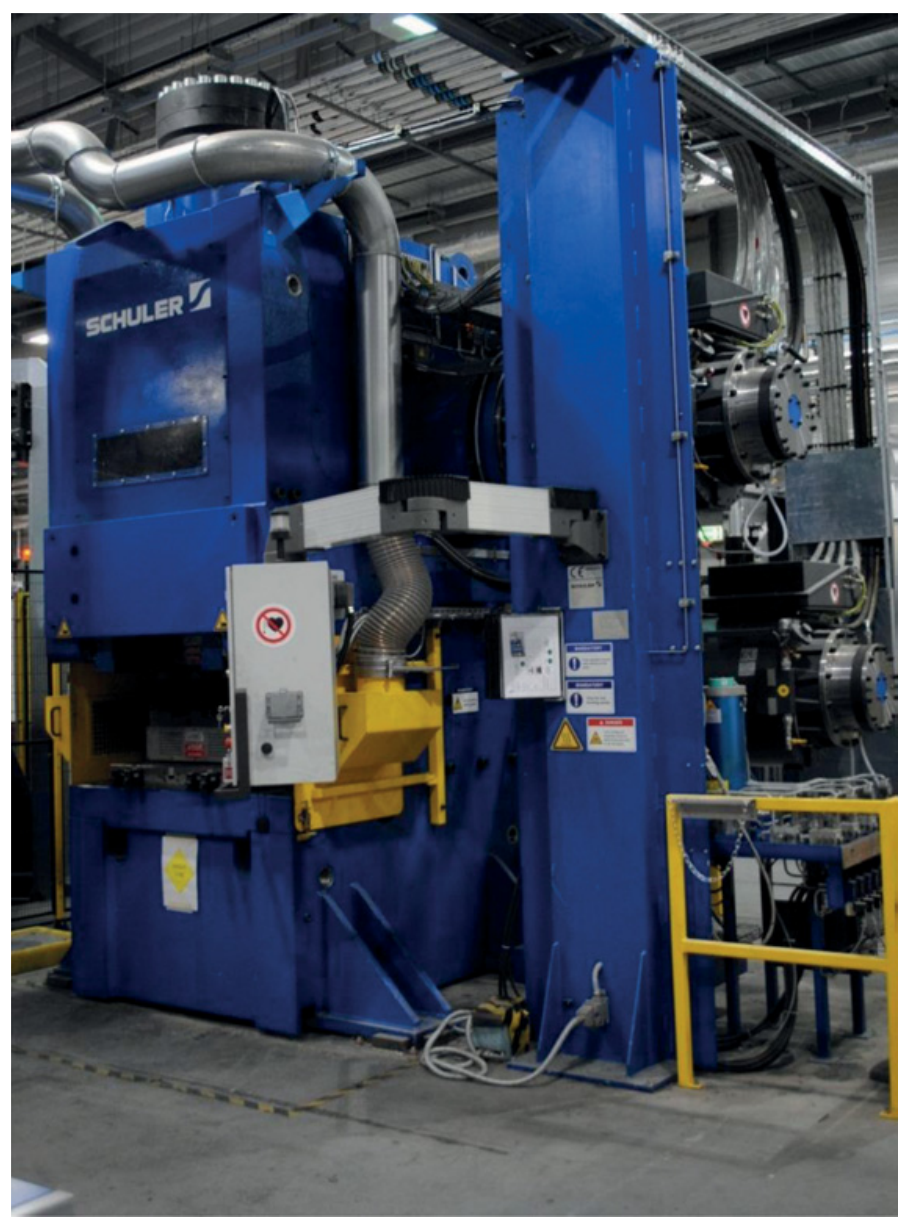

Figure 3. AFRC servo-driven multi-axis press. The motors are just visible on the right hand side of the press.

can understand the process capability and make informed decisions on potential investments in similar equipment. To date, the Centre has won over $£ 2 \mathrm{~m}$ of research linked to this equipment. It is particularly suited to the manufacture of axisymmetric shapes e.g. gears. The processing operation takes place at room temperature and features an upper and lower tool with the upper tool being angled with respect to the lower one in such a way that only a small area of the workpiece is deformed at any point in time. This keeps the overall forming loads low and is one of a number of incremental forming technologies presently being developed by the AFRC. Here, the innovation is not so much the machine itself, rather the overall package of well-developed materials knowledge, process modelling and an ability to produce components to full, or at least meaningful, scale.

Figure 5 shows one of the latest developments in equipment to measure residual stress; the technique uses electron speckle pattern interferometry (Fig. 5a). This machine, manufactured by STRESSTECH, is the first of its type in the UK. The use of a speckle pattern (Fig. 5b) created on the component surface allows the Centre to investigate the residual stress level in areas close to such surfaces. More traditional techniques use strain gauges that have to be glued to the component surface. This necessitates some surface preparation which means that the data from the outer $0.2 \mathrm{~mm}$ of a component is compromised. The STRESSTECH machine relies on interferometry measurements to replace the need for strain 


\section{MATEC Web of Conferences}

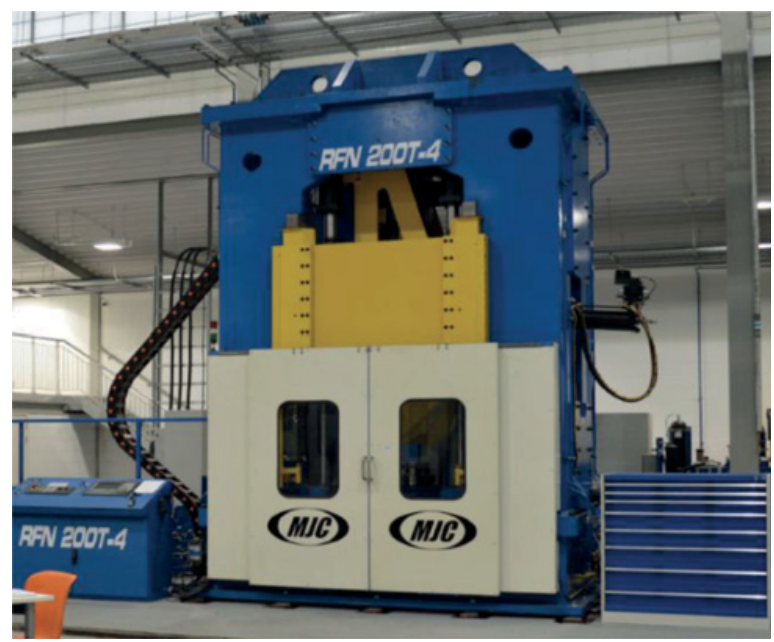

Figure 4. Rotary forging system capable of making parts up to $400 \mathrm{~mm}$ diameter.
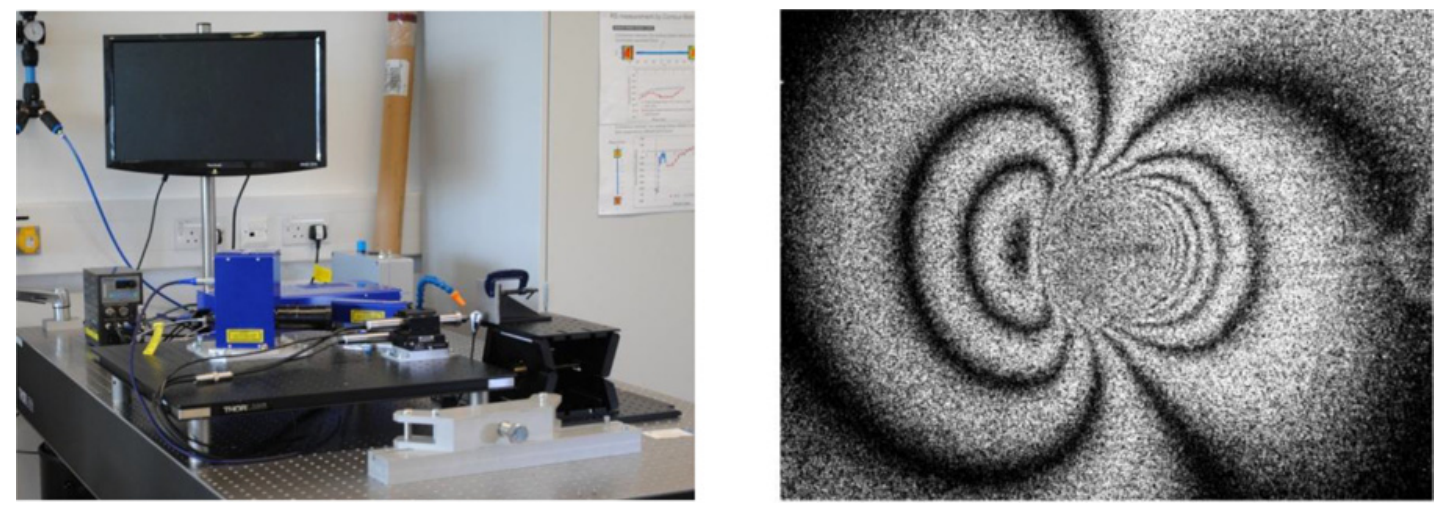

Figure 5. Details of STRESSTECH PRISM residual stress measurement equipment (a) general view (b) typical speckle pattern.

gauges. This technology is one of a number of techniques used within the Centre to develop an understanding of the effects of different processing variables and heat treatments on residual stress distribution. The longer term aim is to develop predictive models to enable residual stress development to be better controlled during production so that problems due to distortion can be minimised.

\section{Modelling validation}

A major element of the offering of Centres such as the AFRC is validated process models. The various requirements of a robust model are listed above. Apart from a good basic knowledge of the workpiece material and a sound modelling approach the final element is the opportunity to validate the model on full scale components; Fig. 6. This latter aspect is important is because it is often difficult to capture 


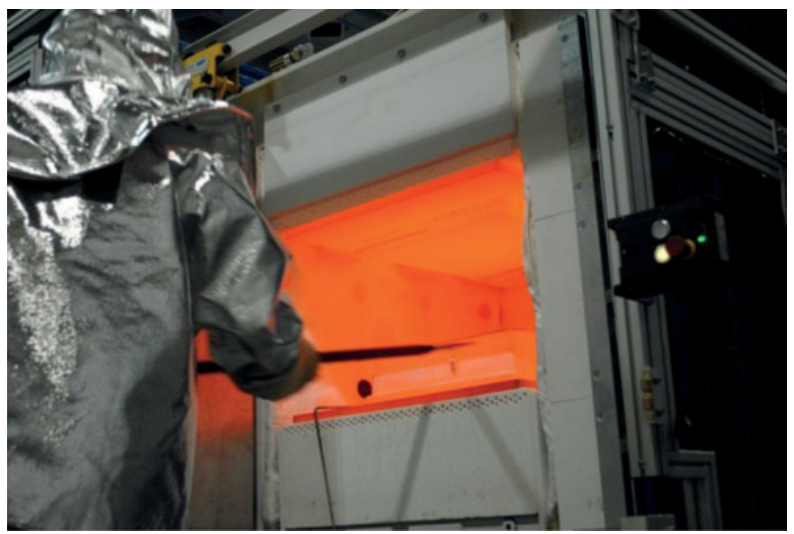

Figure 6. Removal of superplastically formed part from 200 tonne forming press. The forming cycle is developed using a finite element based approach to control the strain rate within the part to be within the optimum forming window.

every aspect of a process in a model e.g. variations in heat transfer rate, friction coefficient or workpiece temperature can have significant effects on component quality. In principle sensitivity analyses can be run using the FE programmes; however the final proof of a model is a successfully formed component. Centres such as the AFRC have industrial sized equipment to enable this final validation stage to be completed. Usually by the time a project has reached this stage it is at the TRL 6 level and the succeeding phase would be transfer of the technology into industry.

\section{Case study}

As an example of how this can work, the AFRC is presently hosting the development of an automated NDE/metrology system to facilitate the measurement and inspection of large scale composite aerostructures for use in aircraft wings [2]. This work, which is set at TRL 4/5 builds on an earlier project completed within the University that established the basic technology up to TRL 3 . The business driver here is to reduce overall cycle inspection times. The intention is that when the present project completes the technology will be transferred into a customer production site.

\section{Summary}

In summary, the role of Catapult centres such as the AFRC are to develop new ideas, or improve preexisting concepts for application into the UK manufacturing base. This may be achieved in a number of ways:

- through working with equipment or technology developers to prove their concepts in a preproduction environment where manufacturers can run trials and de-risk potential investments,

- through the development of validated process models that can be used by manufacturing companies for their own in-house simulations,

- working with alloy designers to both develop new alloys and identify processing windows,

- through the development or improvement of processing technologies that in some cases may be well established, though still capable of improvement, or via the development of new process techniques, 


\section{MATEC Web of Conferences}

Beyond this however, an important role of such Centres is to work with Government to ensure that investment is well directed such that research and development funding targeted at enhancing the performance of the manufacturing sector achieves maximum impact.

\section{References}

[1] H. Hauser; The Current and Future Role of Technology and Innovation Centres in the UK (2010). BIS/Pub/Xk/03/10.NP. URN 10/843

[2] AFRC Highlights; December 2014: https://www.strath.ac.uk/afrc/news/highlightsarchive/ 